\title{
ALternativas FILOSÓficAS, ÉTICAS Y POLITICAS FRENTE A LA CRISIS AMBIENTAL
}

Naína Pierri Estades

\section{Introducción}

uchas presentaciones del problema ecológico actual suponen jue ésta es la primer crisis ambiental o la primera generada por el hombre. Hay detrás una idea de equilibrio natural y de que el hombre sería una especie desnaturalizada, capaz de destruirlo irracionalmente, a pesar de su capacidad racional. Sin embargo, la historia del planeta, jalonada por varias crisis ambientales de orden global (p.e. las generadas por las glaciaciones), desmiente sobradamente el mito del equilibrio natural. Por otra parte, la acción del hombre sobre el medio ha producido, en el pasado, muchas crisis ambientales, sólo que sus consecuencias no pasaron entonces de ser locales o regionales. Por lo tanto, ésta no es la primer crisis ambiental global, ni la primer crisis ambiental generada por el hombre; pero sí sería la primer crisis ambiental global generada por el hombre (Foster, 1994; Ponting, 1992; Foladori, 1999). Esta crisis se caracteriza también por su complejidad, profundidad y posible irreversibilidad. La globalidad, refiere al alcance y dinámica ecosistémicaplanetaria de algunos problemas; la complejidad, al conjunto de dimensiones interrelacionadas e imbricadas de lo socioambiental; la profundidad, a sus causas socialmente estructurales; y la posible irreversibilidad, a que hay aspectos cuya reversión, parece, al menos por ahora, fuera del alcance humano, ya sea debido a su extensión, ritmo, sinergia, gravedad y/o escala temporal de evolución.

Su problematización social y política comenzó a fines de los ‘60s del siglo XX en los países desarrollados y, más adelante, se extendió 
al resto de los. países, aunque con características diferenciadas debido a la existencia de problemas y jerarquías diferentes. La dimensión central de la discusión ha estado puesta en lo económico, y de allí en la creación de instrumentos que orienten el desarrollo para hacerlo compatible con la sustentabilidad ecológica, privilegiándose las soluciones técnicas y los mecanismos de mercado. Pero, nacida de las mismas entrañas de nuestra civilización occidental, esta crisis se lee también como crisis civilizatoria, que cuestiona de forma extrema el mismo proyecto de la Modernidad, poniendo en cuestión los objetivos últimos, los medios y la ética que lo caracterizan. Luego, en tanto la ciencia y la técnica han cumplido un papel clave en la construcción de ese proyecto, pero con resultados extremadamente contradictorios, también son objeto de una profunda revisión. En este artículo, pues, nos proponemos presentar algunas vertientes de la reflexión filosófica, ética y política sobre estos temas, y las principales alternativas planteadas.

Para ello, comenzamos por identificar lo que se entiende por Modernidad y Posmodernidad, diferenciando las épocas históricas de los proyectos de sociedad que en ellas se disputan. Abordamos luego el cuestionamiento contemporáneo a la ciencia clásica y la preocupación por la inversión jerárquica de la ciencia respecto a la técnica, que despoja a la primera de teoría y de la reflexión ética acerca de sus fines. En el capítulo siguiente, abordamos la presentación del problema ambiental que lo plantea como un problema de cómo concebir la naturaleza, si como objeto de uso, que tiene valor en la medida de su utilidad para la sociedad, o como sujeto, con valor intrínseco. Por último, presentamos lo que entendemos como las alternativas políticas fundamentales que se levantan frente a la crisis socioambiental y el "enverdecimiento capitalista" dominante, esto es, la ecología profunda y el ecosocialismo.

\section{Modernidades, malestar contemporáneo, y posmodernidades}

Se parte de constatar un malestar multidimensional en nuestro mundo actual, frente al cual existe la búsqueda de una construcción cultural alternativa. Hablamos de malestares económicos, sociales, éticos, 
políticos, psicológicos, ecológicos, etc., interrelacionados. Y de cultura en el sentido amplio de la palabra, en tanto civilización. Esos malestares suponen un creciente deterioro de los lazos que ligan a las personas entre sí, y también de los lazos que nos ligan con toda trascendencia, sea religiosa o social. Eso supone una pérdida de valores que pasa a legitimar todos los extremos del individualismo y todas las transgresiones. Y en el centro, la paradoja fundamental de una civilización que ha logrado acumular una enorme cantidad de conocimiento, y recursos materiales y técnicos que permitirían resolver no sólo las necesidades sociales básicas, sino tender a liberar a la humanidad de las humillaciones de la desigualdad y la alienación impuestas, pero que están al servicio del lucro y del consumo abusivo de minorías privilegiadas. Luego, esta paradoja aparece culturalmente como una pérdida de significado, de objetivos, de utilidades trascendentes, y en ese sentido, como "apagamiento" o "muerte del hombre" (Foucault, 1966).

Existe, pues, una búsqueda de una episteme nueva (conjunto cultural de teorías y de prácticas, de saberes y de saber-haceres) que permita enfrentar y vencer esos malestares y deshacer esa terrible paradoja. La filosofía contemporánea se pregunta entonces sobre los objetivos de la razón humana, del saber y del saber hacer. En ese sentido, dicho malestar actual suele formularse como crisis de la Modernidad, es decir, del proyecto civilizatorio iniciado en el Renacimiento y desarrollado por la sociedad capitalista hasta el presente. Para ello, la filosofía cuestiona ese proyecto y busca referentes en epistemes anteriores, como la griega, alternativas éticas capaces de promover racionalidades sociales responsables y sentido solidario (p.e. Jonas, Serres, Foucault, Ricoeur, Habermas). Se critica, pues, la Modernidad y se mira el pasado, no en el sentido reaccionario o romántico de idealizarlo y querer retroceder, sino para elaborar una utopía nueva y proponer una ética alternativa, que apunte a superar ese estado de cosas y permita acceder al buen vivir juntos.

Estaríamos viviendo, pues, el cuestionamiento objetivo de la Modernidad planteado por sus resultados paradójicos, y la crítica intelectual o filosófica de sus premisas y de su proyecto. En ese sentido, podría definirse esta época (quizás desde la II Posguerra para acá) como "posmoderna" y, tanto las renuncias al proyecto de la Modernidad, como la búsqueda de una episteme nueva, como "posmodernos". O 
sea, una cosa es el binomio Modernidad-Posmodernidad como identificación de épocas históricas; y la otra es el movimiento filosófico planteado por el "desencanto" o el "fracaso" del proyecto moderno y las búsquedas subsiguientes.

A nuestra manera de ver, tanto la Modernidad como la Posmodernidad no tienen una identidad absoluta, cosa que parece anunciar el hablar de ellas en singular. Cada una tiene una identidad en conflicto, que informa de proyectos alternativos (mutuamente referidos) concebidos en cada época, enfrentados, y relativamente construidos. Es claro que la Modernidad es principalmente el proyecto liberal burgués, que en su lucha por sobreponerse al régimen feudal, levantó las banderas de la libertad, la igualdad y la fraternidad. Proyecto que hizo de la ciencia un instrumento al servicio de la competencia mercantil y la productividad, del saqueo y la dominación bajo todas sus formas. Pero esas banderas, en el vuelo propio de su promesa, y de su incumplimiento burgués, se reformularon casi enseguida en la utopía socialista, de vocación igualitaria y solidaria, y en todas las luchas sociales que pelearon derechos elementales y soñaban con ese orden alternativo. Entonces, no hay un proyecto absoluto de la Modernidad, sino, por un lado, una promesa incumplida por el capital, y, por otro, una utopía pendiente.

Luego, el tiempo de la Posmodernidad, encierra, correspondientemente, la alternativa entre dos grandes maneras de entender el mundo y de posicionarse para actuar en él. Una, la del autodenominado Posmodernismo, el del "vale todo", el del fin de los relatos y la historia, el del individualismo sistemático, el del culto a la indiferencia social y al placer propio, el del cínico desencanto, el antihumanista en su justificación absoluta de la insolidaridad. Y hay, por otro lado, un proceso de reformulación de aquélla utopía, que quiere viabilizarse en un proyecto nuevo capaz de recoger, con afán superador, las duras y tristes enseñanzas de muchas derrotas e impotencias populares, y especialmente las del mal llamado "socialismo real". Proyecto que es antidogmático y, por tanto, diverso y plural; antitotalitario $\mathrm{y}$, por tanto, profundamente democrático; igualitario, aunque no masificador; y claro que también humanista, pero responsable, que valida la posibilidad de la ética social de la responsabilidad. 


\section{El desencanto de la ciencia y la técnica: ¿crisis del conocimiento o de la sociedad?}

En este capítulo, vamos a presentar las dos vertientes de revisión actual de la ciencia y su relación con la técnica. Se trata, en primer lugar, del "hallazgo de la complejidad" que hace a la crisis de la ciencia clásica y prefigura un paradigma alternativo; y, en segundo lugar, de la revisión que se produce frente a la inversión de jerarquía entre la ciencia y la técnica, que supone la transformación de la primera en la llamada "tecnociencia" (Hottois, 1984). El objetivo, además de ordenar cierta información, es precisar el alcance de lo que se critica y de lo que se propone, aportando, en cada caso, nuestro punto de vista.

Uno de los malestares contemporáneos es el "desencanto" frente a la ciencia y la técnica. La filosofía contemporánea, preguntándose sobre los objetivos de la razón humana, del saber y el saber hacer, centra gran parte de su reflexión en la crítica a la Modernidad y sus consecuencias. El leit motiv es señalar los efectos paradójicos de "la Razón", que, de prometer la liberación humana en diferentes terrenos, sólo la genera de manera limitada y, lo que es peor, genera, a la vez, su opuesto: formas de alienación, sometimiento, infelicidad, y hasta destrucción. Se interpreta que la ideología positivista moderna, asignando un valor absoluto al conocimiento científico -hijo dilecto de aquella Razóndejó de lado el cuestionamiento filosófico sobre sus finalidades (Husserl, 1990). Esos efectos perversos, se deberían, entonces, al desgobierno sobre las propias creaciones humanas, generado por la falta de pensar en los fines. La crisis ambiental, desde esa óptica, sería otro efecto perverso y particularmente grave de la cultura moderna en general, y del paradigma científico positivista, en particular. De donde, un cambio de paradigma científico aparece, para quienes comparten esta interpretación, como elemento clave para enfrentarla y superarla.

Nosotros planteamos aquí que esa interpretación mitifica la "Razón" asignándole un sentido genérico que le impide comprender su determinación histórico-social, es decir, le impide ver que adopta fines y formas que dependen de las sociedades concretas en las que se ejerce $y$, particularmente, de los sectores sociales que tienen el poder de subordinar la producción y el uso del conocimiento a sus fines 
particulares. En ese sentido, la cultura moderna es, a la vez, hija e impulsora del desarrollo capitalista. La razón instrumental que la caracteriza, y todos sus frutos (científicos, técnicos, ideológicos, etc.) contribuyeron decisivamente a derribar el orden social anterior y a abrir paso al capitalismo, en su vocación expansiva. Pero claro, los frutos concretos de esa razón histórica guardan todas las paradojas del orden social esquilmante, desigual y desigualador al que sirve, generando posibilidades de liberación de la humanidad (materiales y políticas) que no puede realizar en todo su alcance por el imperio del interés privado sobre el social. Por lo tanto, proponemos que el problema no es esencialmente científico, técnico, filosófico o ético, sino, antes bien, de orden socioeconómico y, por tanto, político.

\section{La crisis de la ciencia clásica frente al hallazgo de la complejidad}

La ciencia, que es vocacionalmente crítica y provisoria, a la vez que construye e implanta un paradigma, sufre procesos de revisión y de creación de nuevas formas de entender el mundo y de ejercerse en él. Es así que, principalmente a lo largo del siglo $\mathrm{XX}$, y desde diferentes vertientes, viene generándose un "redescubrimiento" de lo real que cuestiona las principales premisas teóricas y metodológicas sobre las que se construyó. Se habla, entonces, de la crisis del paradigma científico clásico (mecanicista, positivista, y analítico) y de su paulatina sustitución por uno alternativo, al que se nombra como de la complejidad, y que, unido a la propuesta de una racionalidad social y una ética alternativas, es particularmente promovido por sectores ambientalistas, nombrándolo como "paradigma ambiental".

El paradigma científico clásico reconoce como matriz una serie de ideas o generalizaciones de hallazgos del conocimiento aportados principalmente por grandes pensadores como Francis Bacon (1561-1626), René Descartes (1596-1650) e Isaac Newton (1642-1727), entre otros. Bacon proclamó que "el conocimento es poder" en tanto permitiría a los seres humanos dominar la naturaleza, y trazó ciertas bases metodológicas proponiendo el procedimiento experimental e inductivo para buscar leyes 
generales de orden causal a partir de la observación. Por su parte, Descartes afirmó el dualismo hombre-naturaleza, la separación entre la materia y la mente, lo que, estableciendo la distinción entre objeto y sujeto, habilitó definitivamente la posibilidad del conocimiento científico, pero, a la vez, contribuyó a separar erróneamente al hombre del resto de la naturaleza. Propuso también una visión mecanicista de la naturaleza: animales, plantas y el cuerpo humano funcionarían por reacción mecánica frente a estímulos externos; y una visión del mundo como un orden absoluto donde todo tiene su lugar, lo que permitiría su captación intelectual precisa, formalizable matemáticamente. Consideraba, como dijimos, el conocimiento como medio de convertir al hombre en "Señor de la Naturaleza", es decir, como su poseedor y dominador.

Por su parte, el físico y matemático Isaac Newton formuló la ley de gravitación universal y las leyes de atracción-repulsión de los cuerpos celestes. Su trabajo reafirmó sustantivamente este paradigma, debido al carácter universal de esas leyes, y también por aportar un método matemático que permitía describir el movimiento mecánico, de tal manera que, conociendo el tipo de fuerza y la masa de los cuerpos, bastaba aplicar sus ecuaciones para predecir sus efectos. De hecho, la mecánica newtoniana fue la base de todo el desarrollo posterior de la física mecánica y de sus múltiples y revolucionarias aplicaciones prácticas, desde el siglo XVIII hasta el presente. Luego, en el siglo XIX, este paradigma científico termina de conformarse con la teoría evolucionista de Charles Darwin (1809-1882) que implicaba la idea de un proceso de orden y de progreso, creciente y natural (Novo, 1997; Dobson, 1997; Wagenberg, 1994).

Es pues, en base a esa concepción del mundo y de la misma ciencia, que tuvo lugar la extraordinaria producción de conocimiento que ha servido de base al avance tecnológico que llega a nuestros días, proceso que, por bastante tiempo parecía confirmar que el dominio racional de las leyes que rigen la naturaleza sería el camino del progreso, entendido como artífice del bienestar general. La también extraordinaria generación y acumulación de efectos perversos de ese progreso -de la cual la crisis ambiental actual es una manifestación elocuente- resulta un profundo cuestionamiento que alimenta la revisión profunda del sentido de la ciencia y de las formas en que ella se traduce en resultados sociales. 
Las dudas sobre la validez absoluta de este paradigma se fueron expresando, por lo menos, desde el siglo XIX, por filósofos y cientistas sociales. Pero ganaron mayor peso las provenientes del campo de las ciencias físicas y las matemáticas, primero, y luego, de la biología, y la ecología, en particular, a partir de una serie de hallazgos. Y fue recién en los años ' 60 s del siglo XX, que el malestar acumulado debido a la incapacidad de los presupuestos newtonianos para resolver problemas relacionados con fenómenos cada vez más complejos, hizo eclosión y tomó estado público. Pero debe entenderse que no es que la física newtoniana esté equivocada; sino que los sistemas estables y reversibles en el tiempo que ella describe, son sólo una parte de la realidad: p.e. describe el movimiento de los planetas, pero no el desarrollo del sistema planetario. Su validez está limitada a los sistemas en equilibrio o próximos al mismo, que es donde, conociendo la ley y las condiciones iniciales, es posible determinar sus estados futuros. Pero en los sistemas que están lejos del equilibrio -que son tantos o más que los anteriores- existe un desarrollo "constructivo" en el tiempo por el que el futuro es incierto y las condiciones son irreversibles. Por eso, las leyes que pueden enunciarse para esos casos, apenas enumeran posibilidades. Es así que, actualmente, las ciencias naturales intentan formular leyes capaces de captar la irreversibilidad y expresar la probabilidad, esperando compatibilizar la idea de leyes de la naturaleza con los eventos, la creatividad y la innovación que se observan en ella (Bertalanffy, 1968; Murphy \& O’ Neill (orgs.), 1997; Wallerstein, 1996; Wagenberg, 1994).

Estamos, pues, frente a una crisis de las explicaciones simples y deterministas de un mundo que se veía ordenado. La incertidumbre, el desorden, la contradicción, la pluralidad, la complicación, que aparecían como residuos no científicos, hacen hoy a la problemática general de la ciencia. Las ideas de interdependencia, probabilidad, indeterminismo y desorden confluyen en atisbar la complejidad real del mundo y en mostrar los límites que tiene el paradigma mecanicista para dar cuenta de él. Va surgiendo, entonces, un nuevo paradigma "de la complejidad", y que, según elementos que se le agreguen o se destaquen, se nombra también como "sistémico", "holístico", "ecológico", o "ambiental" (Bertalanffy, 1968; Prigogine, 1997; Morin, 1990 y 1994; Novo, 1997). 
Quiere decir que un conjunto de nuevos conocimientos componen una nueva percepción de la realidad, captada ahora como compleja, y esto revierte en la propia ciencia removiendo algunos de sus supuestos, objetivos y métodos básicos, a la vez que permite inferir otros, que se supone que la harían capaz de aproximarse mejor a dar cuenta de esa complejidad. Ahora, cuando hoy se habla de "complejidad" se habla principalmente del reconocimiento -más o menos fundado o preciso- de la existencia de lo complejo y del proceso de divulgación y asunción social de esa nueva visión del mundo. Ya como paradigma científico que debería superar al mecanicista, las cosas son más precarias: hay advertencias y recomendaciones, más que teorías, métodos o procedimientos decididamente alternativos a la ciencia clásica (Wagenberg, 1994). Y cabe aquí la pregunta de qué tradición filosófica y teórica, o qué propuesta nueva, se acerca más a describir y explicar la complejidad. Nosotros entendemos que la captación del movimiento de la naturaleza, en general, y de la sociedad, en particular, alcanzada mediante la conjunción de la dialéctica y el materialismo histórico, ya desde mediados del siglo XIX, daba cuenta de gran parte de lo que hoy se encierra bajo la idea de complejidad. Y que, desde el punto de vista teórico-metodológico, esa tradición sofistica y califica la propuesta actualmente más promovida de adoptar un enfoque sistémico, porque éste es de por sí formal, vaciado de teoría, y no necesariamente dialéctico.

Desde esa concepción, es que interpretamos que es erróneo atribuir la responsabiliad del malestar contemporáneo, en general, y de la crisis ambiental, en particular, al paradigma científico dominante o a la falta de discusión ética sobre los fines de la ciencia y sus aplicaciones técnicas. Decimos esto no porque dudemos de la necesidad y oportunidad de superar los límites del primero, y de dar lugar a esa discusión, sino porque entendemos que la causa de fondo de esta gran crisis civilizatoria radica en la lógica privada que preside la sociedad de mercado, donde ciencia y técnica operan como medios privilegiados de su reproducción. Criticar la ideología positivista, que las consagró como diosas de la Modernidad, es muy importante para devolverle a la sociedad el derecho de cuestionar su subordinación a los intereses de minorías privilegiadas. Pero abonar esa crítica creyendo que una nueva ciencia supondría la vía privilegiada de superación de la crisis, invierte los 
- términos de la realidad, impidiendo la percepción del carácter social del problema y la identificación de sus responsables, esto es, su politización, con lo que, en realidad, contribuye a su persistencia. Decimos entonces que el gran desafío no es principalmente cambiar el paradigma científico, ni impregnarlo de otra ética, sino sustituir el orden social presidido por el capital, por otro, que atienda las necesidades sociales de las mayorías. Luego, será en función de ese nuevo orden que se podrá recuperar el sentido fundamentalmente progresivo de la producción y aplicación del conocimiento científico.

\section{De la ciencia a la "tecno-ciencia" o el abandono de la teoría y de la ética}

Ocupémonos ahora del segundo aspecto del cuestionamiento contemporáneo a la ciencia, dado por la evolución del sentido y el cambio de jerarquía entre ciencia y técnica.

La especificidad de nuestra especie es que organizamos nuestra sobrevivencia y reproducción mediante el trabajo social basado en la fabricación de instrumentos, relativamente independientes de su uso inmediato. Eso supone un proceso de acumulación y objetivación de información expresado en la evolución técnica, que durante mucho tiempo no supuso ciencia, teoría, sino manipulación y ensayo (bricolage). Quiere decir que en la historia de la humanidad, la técnica precedió a la ciencia, el saber-hacer al saber. Por su parte, el nacimiento y desarrollo de la ciencia moderna, aunque no es independiente del desafío técnico planteado por las necesidades de la producción, supuso una jerarquización del momento teórico y abrió un epacio de reflexión acerca de los fines sociales de sus aplicaciones.

Contemporáneamente, frente al carácter contradictorio o perverso de muchos de las aplicaciones del conocimiento científico, se observa que paulatinamente se ha producido un cambio de jerarquía entre la ciencia y la técnica, esto es, entre la teoría y la práctica, entre el saber y su utilización, entre la reflexión y la acción, que explicaría la pérdida de su signo fundacional humanista y progresista. Se propone, así, que en el siglo XX la ciencia se ha transformado en "tecno-ciencia" (Hottois, 
19834), palabra que quiere dar relevancia al hecho de que su ejercicio tiene cada vez menos teoría y cada vez más técnica. Lo que la ciencia busca no es más un conocimiento que sirva al bienestar humano, con fines técnicos diversos, elegibles según la realidad social y la reflexión. Avanza según lo que es técnicamente posible hacer, sin teoría ni reflexión, jalonándose en sucesivas sofisticaciones, y se justifica en esa dinámica alienada de humanidad, en vez de hacerlo a través de saberes con fines y sentido humano.

A nuestra manera de ver, este planteo adolece del mismo problema que el anterior: separa a la ciencia de la dinámica social en la que se inscribe, que es lo que la aliena de su sentido potencialmente progresivo, y la desliza a desinteresarse de su significado social y ético, a la vez que la vuelve una ideología autojustificativa del orden social al que sirve (Habermas, 1986). Luego, también fetichiza y demoniza inadecuadamente la técnica, como si tuviera vida propia y ella en sí fuera responsable de las formas y uso que la sociedad le da. Entendemos que ni la ciencia ni la técnica son buenas o malas, genéricamente. Y que, si bien el conocimiento teórico y técnico son necesarios para el progreso y el bienestar humanos, son de por sí insuficientes, porque están fundamentalmente subordinados a la forma de organización social imperante y destinados a su reproducción, más que a su crítica y transformación..

\section{La naturaleza como objeto, sujeto, o categoría histórico-social}

La crisis ecológica actual ha puesto en primer lugar la reflexión sobre la relación hombre-naturaleza. Respecto a ella, somos herederos de dos concepciones: la griega y la hebraica. La primera, plantea que todo lo que existe, incluyendo el hombre, pertenece a la naturaleza. Esta tiene una razón de ser y el hombre en ella, el que, en función de su razón, puede conocerla. Por su parte, la concepción hebraica, enticnde que la Palabra-Dios es exterior a toda la naturaleza. Esa naturaleza fue creada por Dios, al igual que el hombre, que es parte de ella. Pero el hombre, creado a imagen y semejanza de Dios, puede trasnsformarse en Señor de la naturaleza, es decir, crearla y dominarla. 
Periodizando la historia de la humanidad según su concepción de la relación con la naturaleza, podemos distinguir tres culturas sucesivas:

- la antigüedad, que, desde la concepción griega, creía que el hombre no podía dominar la naturaleza, sino acompañarla, obedecerla y hasta adorarla;

- la época moderna, adscripta a la concepción hebraica, que entendía al hombre como poseedor y dominador de la naturaleza (Descartes, Bacon, Kant, Hegel, etc.). Esto es, que a través de la razón y la fabricación puede poseer y dominarla intelectual y técnicamente; y

- la cultura contemporánea que, frente a las consecuencias negativas de esa dominación, estaría retornando a una concepción monista, buscando referentes en los antiguos para reorientar la relación hombrenaturaleza hacia una sustentabilidad socioambiental.

Las alternativas de cómo concebir la naturaleza o la relación con ella que hoy parecen existir, se presentan como concepciones filosóficas y prácticas dentro del binomio naturaleza-objeto versus naturaleza-sujeto. Por un lado, persistir en considerar la naturaleza como objeto, cosa, pura materia y espacio, externa y hasta opuesta al hombre, con la cual éste se relaciona más cuantitativa que cualitativamente, la naturaleza como simple reserva de recursos y depósito de basura, la naturaleza, en fin, como objeto de uso, potencial o real. $\mathrm{O}$, por otro, considerarla sujeto, fuerza de vida, múltiple y contradictoria (buena y mala), en la que vivimos los humanos, y según la cual tenemos que vivir, y que, en tanto sujeto, tiene derechos, es amable, es respetable. Estas alternativas expresarían, pues, el drama ético actual frente a la crisis ecológica.

Lo anterior puede expresarse también como alternativa entre un antropocentrismo irresponsable e instrumentalista, que preside la sociedad en que vivimos, y un ecocentrismo que parece reconocer la humanidad como parte de la naturaleza, pero que subordina los derechos humanos a los derechos de ésta. Este ecocentrismo parte de la visión del hombre malo, separándolo y oponiéndolo a una naturaleza idealizada. Posee una captación del hombre como especie biológica, sin conseguir considerar la especificidad de su estatuto social, que es la forma en que la especie existe. Luego, esto induce a una identidad especie-sociedad 
humana que extrapola a ésta una homogeneidad falsa. En primer lugar, suponiendo que los hombres habrían tenido siempre la misma actitud con el medio, reconociendo sólo una evolución basada en el crecimiento demográfico y la superación técnica. En segundo lugar, suponiendo una homogeneidad social interna según la cual todos los miembros de la sociedad gozarían de los mismos derechos de uso de los recursos naturales, uso en el que serían igualmente beneficiados y/o perjudicados. Una variante de esta visión del hombre malo es fraccionar la trayectoria de la humanidad en dos: un pasado inocente y/o sabio en el que el hombre vivía en armonía con la naturaleza (que se extiende a los pueblos primitivos y las comunidades campesinas actuales); y una etapa posterior, en la que se habría convertido en egoísta y destructivo, generalmente asociada con el desarrollo tecnológico industrial y la vida ciudadana (Dobson, 1997; Foladori, 1999).

A nuestra manera de ver, tanto el antropocentrismo irresponsable dominante como el ecocentrismo, se elaboran desde una falacia común, que es entender la naturaleza y la socieadad humana como cosas diferentes, y hasta opuestas, de donde problematizan sus relaciones y se colocan como defensores absolutos de una u otra. Frente a esto, entendemos que la alternativa sustantiva es concebir la sociedad como una parte de la naturaleza que tiene la especificidad de ser no sólo objeto sino sujeto de su propia transformación. Y proponemos que para entender cómo la sociedad se relaciona con el resto de la naturaleza hay que superar la visión biologicista y ahistórica que preside el ecocentrismo y buscar su explicación en las leyes sociales históricamente determinadas, que, en un sentido, cumplen, y en otro se sobreponen, a las leyes naturales. Luego, la naturaleza, porque incluye al hombre en su especificidad, está socialmente determinada, inclusive donde aparece "intocada". Y por eso decimos que, siendo a la vez sujeto y objeto, tiene un estatuto de categoría histórico-social, que adopta formas concretas, determinadas por las relaciones sociales prevalecientes cada vez (Foster, 1994; Foladori, 1999).

Más concretamente, nos referimos a que la historia de la humanidad reconoce etapas cualitativamente distintas, establecidas por diferentes formas de organización social de la producción, que han supuesto diferentes modalidades de apropiación social y uso económico de la restante naturaleza. En los casos en que esos modos de producción 
- se organizaron en base a relaciones sociales comunitarias e igualitarias, el vínculo con ella, tendió a ser armónico, más allá de los resultados desconocidos y/o no queridos de toda operación técnica. Por el contrario, en los casos en que esas relaciones fueron de explotación y dominación en base a la apropiación privada de los medios productivos, ha sido y es esa misma modalidad de relación la que se proyecta hacia la restante naturaleza. Luego, las diferencias de clase, suponen un acceso diferencial a los recursos naturales, que establece responsabilidades distintas respecto a la forma social de utilizarlos y un reparto muy desigual de beneficios y perjuicios, que es lo que alimenta el carácter profundamente social y conflictivo de todo problema ambiental. Por lo tanto, el interés humano en la restante naturaleza, como ámbito de vida y fuente de recursos para satisfacer sus necesidades, si bien tiene un sustrato inicialmente biológico, como en todas las especies vivientes, adopta formas históricas y sociales concretas que, en las sociedades clasistas, son de clase (Ponting, 1992; Foster, 1994; Foladori, 1999).

En síntesis, frente a la forma tradicional de presentar esta cuestión naturaleza- objeto versus naturaleza-sujeto, estamos proponiendo la alternativa de considerar la naturaleza -que comprende la sociedad-, a la vez y dialécticamente sujeto y objeto, que adopta formas concretas, determinadas por las relaciones sociales. En ese sentido, considerarla como categoría histórico-social. Y también proponemos que esta forma de concebir la naturaleza como socialmente determinada y la sociedad como parte de ella, es el espacio más adecuado para albergar la tercer alternativa ética, aquélla del antropocentrismo o humanismo responsable (p.e. Jonas; Habermas), porque comparten una visión histórico-social crítica, recogiendo las promesas no cumplidas de la Modernidad, y colocándose del lado de los desfavorecidos por ella.

\section{Las grandes opciones éticas y políticas frente a la crisis ambiental actual}

Frente a la crisis ambiental actual se han elaborado diferentes propuestas políticas para su superación. Por un lado, asistimos a la concepción dominante, ética, teórica, política e instrumental que, desde 
una limitada voluntad de "enverdecimiento" confirma, más que lo que cambia, el orden socio-ambiental actual. Frente a esta realidad, se levantan dos grandes alternativas que, priorizando uno $\mathrm{u}$ otro de los términos del binomio naturaleza-sociedad, postulan éticas opuestas y caminos políticos sensiblemente diferentes, aunque esto cae en el terreno mucho más amplio de cómo transformar las identidades fundamentales de una sociedad. Se trata, por un lado, de la llamada "ecología radical" o "deep ecology", y, por otro, del "ecosocialismo". El objetivo de este apartado es, pues, describir cada uno y discutir un poco el proyecto de sociedad que tienen en su horizonte.

La "ecología radical" es un movimiento cultural, de carácter ecocentrista, cuyos ideólogos provienen de diferentes campos: naturalistas, como Aldo Leopold; filósofos, como Arne Naess, Bill Devall, y George Sessions ${ }^{1}$; cientistas, como J. Lovelock y Carson; teólogos, como Nash; juristas, como C. Stone; utopistas, como A. Huxley y Shepard, periodistas, como Muir; poetas, como Thoureau y Snyder. La "biblia" de ese movimiento es el libro de Aldo Leopold (1949), A sound county almanac and sketches here and there, donde propone una ecosofía y una ética de la Tierra por la cual todos los individuos deberían sufrir una conversión mental y de comportamiento capaz de permitir que el hombre se coloque en armonía con la naturaleza. Para ello, plantea tres grandes orientaciones:

- pensar en términos holistas, biosféricos, lo que supone dejar de considerar al hombre en el centro de un ambiente que lo rodea, y pensarlo como una parte más de un todo viviente;

- pasar del antropocentrismo al biocentrismo, que es colocar la Vida en el centro, como valor supremo. Esto implica, por un lado, que todos los elementos de la naturaleza poseen valor intrínseco, y merecen una ética de conservación. Y, por otro, que todos los seres vivos son iguales respecto al derecho a la vida, de donde, en caso de conflicto entre los derechos del hombre y los de la restante naturaleza, la preferencia debe ser por los derechos de ésta; y

- cultivar la ecosofía, religión de la naturaleza por la cual las personas se proponen adoptar la sabiduría de la Tierra. Esto supone, por ejemplo, practicar el culto del cuerpo, preservar a las mujeres (por considerar 
que están más próximas a los ritos de la naturaleza), preferir la alimentación vegetariana, respetar la biodiversidad, valorizar los modos de vida de los pueblos y culturas humanas en extinción, etc..

Otro elemento importante es que frente a la crisis ambiental actual, uno de cuyos componentes es la explosión demográfica, la valoración jerárquica de la restante naturaleza, conduce a esta concepción a proponer una drástica disminuición de la población humana, aunque no especifican los medios para llegar a ella, ni quiénes serían las sociedades o sectores sociales que deberían ceder su espacio a las otras especies y a la minoría humana privilegiada con el derecho de vivir. Por último, la propuesta política no pasa por incentivar la participación en las comunidades, sino por desarrollar una "ciudadanía biótica" individual, esto es, que cada persona adopte para sí y para los otros, un comportamiento armónico con la naturaleza.

Vayamos ahora a una somera presentación de lo que llamamos "ecosocialismo". En este caso, no se trata de un movimiento cultural sino de un proyecto político (en el sentido más amplio de la palabra, y por tanto, también cultural), con diversas vertientes y que no tiene un manifiesto que sintetice sus ideas fundamentales. En él convergen las distintas tradiciones teóricas fundadas en una crítica radical al capitalismo, esto es, principalmente el marxismo ${ }^{2}$ y el anarquismo comunitario ${ }^{3}$, así como proyectos fuertemente reformistas como los popular-democráticos, los populismos campesinistas ${ }^{4}$ y el llamado "ecologismo de los pobres"5. Y también, movimientos culturales como el feminismo ${ }^{6}$, profundo cuestionador de los órdenes de dominación que, articulados con el poder del capital, atraviesan toda la dinámica social. Por último, podrían considerarse también dentro de esta corriente crítico-propositiva las movilizaciones sociales recientes que se autoidentifican en contra de la globalización capitalista, y reclaman la "justicia global", protestando contra el injusto y esquilmante orden comercial mundial (Seattle, 1999), y contra el papel "imperialista" del Banco Mundial y el Fondo Monetario Internacional (Wáshington, abril/2000) (Bañales, 2000.a y b).

Lo que justifica considerarlos bajo un proyecto común, además de la crítica profunda al orden actual, es que, aún en su heterogeneidad, viajan en la corriente de todas las luchas populares de la Modernidad 
no agotada y validan como objetivos colectivos sus promesas incumplidas, absteniéndose del desencanto posmoderno individualista. Es, en ese sentido, un proyecto contra toda forma arbitraria de poder, contra toda forma injusta de desigualdad, contra toda lógica de insolidaridad social. Y tiene por base la confianza en la posibilidad de la responsabilidad social; no en la omnipotencia técnica, ni en las administraciones bien intencionadas de sabios o de tecno-burócratas.

En esta amplia confluencia, los caminos de la política tampoco son únicos, pues cada vertiente teórica y cada movimiento tiene propuestas y ensayos particulares. Destacamos aquí una propuesta teórica que goza de una adhesión bastante amplia en muchos sectores críticos, la de la acción comunicativa de Habermas (1987). Filósofo y sociólogo de la Escuela de Frankfurt, Habermas propone una ética comunicacional, una ética de la discusión, partiendo del hecho de que la palabra dialógica es normativa. La idea es que el ejercicio de la comunicación social, crea la misma sociedad, disputa sentidos y establece normas. Pero, para que esa acción comunicativa pueda ejercerse en el sentido de las transformaciones deseadas, son necesarias ciertas condiciones políticas, culturales y sociales. La condición política, es la más amplia democracia; la condición cultural, reinstaurar el valor de la discusión y volverla verdaderamente pública; y la condición social, que las personas sean económicamente iguales frente a la discusión. O sea, se trata de una propuesta de socialización máxima de los medios de información, de los espacios de discusión, y de los medios de producción. Por ello, esencialmente revolucionaria, aunque de procesamiento gradual y reformista.

Quiere decir que, frente a la crisis socioambiental actual y al enverdecimiento capitalista dominante, existen dos grandes opciones filosófico-éticas y políticas. Por un lado, la de la ecología radical, biocentrista, antihumanista, que privilegia la conservación de la vida no humana a costa inclusive de la misma humanidad, y cuya propuesta política es la conversión individual hacia una comunión de cada uno con la diversidad biosférica. Se trata de una especie de anarquismo individualista que no ofrece una alternativa real a esta sociedad, sino, en el mejor de los casos, una aggiornada religión de la naturaleza. Y, por otro lado, un proyecto ecosocialista, profundamente humanista, heredero de las mejores luchas y objetivos de la Modernidad, plural y democrático, 
y que apuesta a los caminos colectivos para establecer una sociedad solidaria y responsable de sí misma, que es ser, al mismo tiempo, responsable con la restante naturaleza.

\section{Conclusiones}

La crisis ambiental actual se lee también como crisis civilizatoria porque cuestiona de forma extrema el mismo proyecto de la Modernidad, poniendo en cuestión sus objetivos, los medios y la ética que lo caracterizan. Luego, en tanto la ciencia y la técnica han cumplido un papel clave en su instrumentación pero con resultados extremadamente contradictorios, también son objeto de una profunda revisión. En este artículo nos propusimos presentar algunas vertientes de la reflexión filosófica, ética y política sobre estos temas, y las principales alternativas planteadas.

Para ello, comenzamos por identificar lo que se entiende por Modernidad y Posmodernidad, diferenciando las épocas históricas de los proyectos de sociedad en ellas emergidos. Propusimos que cada época, si bien tiene un proyecto dominante, alberga otro que se le contrapone y que recoje los objetivos sociales incumplidos y las utopías pendientes. Actualmente, frente al cínico e individualista desencanto posmoderno, se levanta un proyecto que apuesta a la posibilidad de recuperar la ética de la responsabilidad social, que incluye lo ambiental.

Abordamos luego el cuestionamiento contemporáneo a la ciencia clásica y la preocupación por la inversión jerárquica de la ciencia respecto a la técnica. Planteamos allí que la interpretación, bastante extendida, de adjudicarle al paradigma científico positivista la responsabilidad por la racionalidad individualista e instrumental dominante es invertir los órdenes de causalidad desde una concepción idealista, ahistórica y asocial. Propusimos, entonces, que el control de la producción del conocimiento y el uso de sus aplicaciones por parte del capital, es lo que subordina los productos históricos de la razón a su lógica privada poniéndolos en contradicción con el progreso y el bienestar sociales. Y que el problema no se resuelve cambiando el paradigma científico, ni impregnándolo de otra ética -aunque ambas 
cosas sean de por sí positivas-, sino sustituyendo el orden social actual por otro que atienda las necesidades sociales de las mayorías.

En el capítulo siguiente, abordamos la formulación del problema ambiental que lo plantea como un problema de cómo concebir la naturaleza, si como objeto de uso, que tiene valor en la medida de su utilidad, o como sujeto con valor independiente, esto es, valor intrínseco. Propusimos que ese planteo es falaz por separar y oponer erróneamente sociedad y naturaleza, y que hay una tercera alternativa que, partiendo de considerar la sociedad como parte de la naturaleza, con una capacidad particular de transformarla y transformarse, ésta es objeto y sujeto a la vez, y su carácter, por tanto, es histórico-social. Interpretamos también que detrás del binomio inicial están, respectivamente, las posiciones filosóficas del antropocentrismo utilitarista dominante y del ecocentrismo.

Por último, presentamos lo que entendemos como las alternativas políticas fundamentales que se levantan frente a la crisis socio-ambiental y el "enverdecimiento capitalista" dominante: la ecología profunda y el ecosocialismo, identificando sus sustratos filosófico-éticos. Propusimos allí que el ecosocialimo es la opción contemporánea que recoge las mejores banderas de las luchas sociales de la época moderna, y que, desde el antropocentrismo responsable, es el depositario más legítimo de la utopía pendiente.

\section{Notas}

${ }^{1}$ La plataforma aparece en Naess, 1986; o en Devall, \& Sessions, G., 1985, Deep ecology: living as if nature mattered, Gibbs Smith, Salt Lake Ciy, Págs. 69-73.

${ }^{2}$ Véase, por ejemplo, dentro de la vertiente marxista en sentido amplio: Marcuse, Herbert, [1979] 1993, La ecología y la crítica de la sociedad moderna, Ecología Política N $^{\circ}$ 5, Abril/93, Págs. 73-79, Barcelona; Coates, Ken (ed.), 1979, Socialism and Environment, Spokesman, Nottingham; Gorz, André, 1991, Capitalisme, Socialisme, Ecologie, Galileo, Paris; Foster, John Bellamy, 1994, The vulnerable planet, Monthly Review Press, New York; O'Connor, James, 1991, Las condiciones de producción. Por un marxismo ecológico, una introducción teórica, Ecología Política $\mathrm{N}^{\circ}$ 1, Abril/91, Págs. 113-130, Barcelona; Leff, Enrique, 1994, Ecología y capital. Racionalidad ambiental, democracia participativa y desarrollo sustentable, Siglo XXI, México 
${ }^{3}$ Véase, por ejemplo: Masjuan, Eduard, 1993, Población y recursos naturales en el anarquismo ibérico: una perspectiva ecológico-humana en el marco del "Socialismo de los pobres"; Parte I, en Ecología Política $\mathrm{N}^{\circ}$ 5, Abril/93, Págs. 41-55; Parte II en Ecología Política $\mathrm{N}^{\circ} 6$, octubre/93, Págs. 129-152, Barcelona

${ }^{4}$ Véase, por ejemplo, González de Molina, Manuel \& Sevilla Guzmán, Eduardo, 1992, Una propuesta de diálogo entre socialismo y ecología: el neopopulismo ecológico, en Ecología Política $\mathrm{N}^{\circ}$ 3, Abril/92, Págs. 121-135, Barcelona.

${ }^{5}$ Véase, por ejemplo: Martínez Alier, Joan, 1995.

${ }^{6}$ Véase, por ejemplo, Salleh, Ariel, 1991, Ecosocialismo-Ecofeminismo, en Ecología Política $\mathrm{N}^{\circ}$ 2, Set.191, Págs. 89-92, Barcelona; Salleh, Ariel, 1994, Naturaleza, mujer, trabajo, capital: la más profunda contradicción, en Ecología Política $\mathrm{N}^{\circ} 7$, abril.194, Págs. 35-47, Barcelona; Mellor, Mary, 1997, Un socialismo verde y feminista: la teoría y la práctica, en: Ecología Política $\mathrm{N}^{\circ} 14$, abril/97, Págs. 11-22, Barcelona.

\section{Referências Bibliografícas}

BAÑALES, Jorge. Movimientos sociales contra el FMI. Venid y vamos, todos. Brecha Año 15, $\mathrm{N}^{\circ} 749,7 / 4 / 2000$, Págs. 28-29, Montevideo, 2000.

Voces y acciones contra la globalización. El dragón y la tortuga. en Brecha Año 15, $\mathrm{N}^{\circ}$ 751, 20/4/2000, Pág. 28, Montevideo, 2000.

BERTALANFFY, Ludwig von. Teoria geral dos sistemas. Vozes, Petrópolis, 1968.

DOBSON, Andrew. Pensamiento político verde. Una nueva ideología para el siglo XXI. Paidós, Barcelona, 1997.

FOLADORI, Guillermo. Los límites del desarrollo sustentable. EBO-Trabajo y capital, Montevideo, 1999

FOSTER, John Bellamy. The vulnerable planet. A short history of the environment. Monthly Review Press, New York, 1994.

FOUCAULT, Michel. Les mots et les chosses, Gallimard, Paris, 1966.

GÓMEZ-HERAS, José M.G. (coord.) Ética del medio ambiente. Problema, perspectivas, historia. Tecnos, Madrid, 1997.

HABERMAS, Jurgen. Ciencia y técnica como "ideología". Tecnos, Madrid, 1986. 
Madrid, 1987.

Teoría de la acción comunicativa. Volumen I y II, Taurus,

HOTTOIS, Gilbert. Le signe et le technique. Aubier, Paris, 1984.

HUSSERL, Edmond. La crise de l'humanité européenne et la philosophie. Hatier, Paris, 1990.

JONAS, Hans. El principio de responsabilidad. Ensayo de una ética para la civilización tecnológica. Herder, Barcelona, 1995.

LEOPOLD, Aldo. A sound county almanac and sketches here and there. University Press, Oxford-London, 1968.

MARCUSE, Herbert. El hombre unidimensional. Planeta-Agostini, Barcelona, 1985

MARTÍNEZ Alier, Joan. De la economía ecológica al ecologismo popular: Nordan-Icaria, Montevideo, 1995.

MORIN, Edgar. Ciencia con conciencia. Biblioteca Universitaria, Portugal, 1990. La complexité humaine, Flammarion, París

MURPHY, M. \& O'Neill, L. (orgs.) "O que é vida" 50 anos depois. UNESPCambridge University Press, Sao Paulo, 1997.

NAESS, Arne. The shallow and the deep, long-range ecological movement. Inquiry, No 16, Págs. 95-100, 1973.

The deep ecology movement: some philosophical aspects. Philosophical Inquiry, $\mathrm{N}^{\circ}$ 8, Págs. 10-31, 1986.

NOVO, María La educación ambiental en el marco del paradigma ambientalista, en Máster en Educación Ambiental, Colección de Monografías $\mathrm{N}^{\circ} 10$, págs. 7 41,UNED-FUE, Madrid, 1997.

PONTING, Clive. Historia verde del mundo, Paidós, Barcelona, 1992.

PRIGOGINE, Ilya. O fim das certezas, Editora UNESP, Sao Paulo, 1997.

WAGENSBERG, Jorge. Ideas sobre la complejidad del mundo, Tusquets, Barcelona, 1994.

WALLERSTEIN, Immanuel. Para abrir as ciencias sociais, Cortez, Sao Paulo, 1996. 


\section{Resumen}

La crisis ambiental actual se lee también como crisis civilizatoria porque cuestiona de forma extrema el mismo proyecto de la Modernidad, poniendo en cuestión sus objetivos, los medios y la ética que lo caracterizan. Luego, en tanto la ciencia y la técnica han cumplido un papel clave en su instrumentación, pero con resultados extremadamente contradictorios, también son objeto de una profunda revisión. En este artículo presentamos algunas vertientes de la reflexión filosófica, ética y política sobre estos temas, y las principales alternativas planteadas

Palabras-clave:crisis ambiental; ética; política

\section{Resumo}

A crise ambiental atual lê-se também como uma crise civilizatória pois questiona, de forma extrema, o próprio projeto da Modernidade, colocando em questão seus objetivos, os meios e a ética que o caracterizam. Assim, a ciência e a técnica têm cumprido um papel chave em sua instrumentalizacão, mas com resultados extremamente contraditórios e sujeitos a uma profunda revisão. Neste artigo apresentamos algumas vertentes da reflexão filosófica, ética e política sobre estes temas, e também as principais alternativas colocadas.

Palavras-chave: crise ambiental; ética; política.

\section{Abstract}

The present environmental crisis is also read as a civilizatory crisis, because it questions deeply the Modernity project, its objectives, means and ethics. While science and technics played a central role in its instrumentalization, it also lead to extremely contradictory results, which makes them object of a deep revision. In this paper, we present some philosophical, ethical and political reflections on the topic, and the main alternatives in place.

Key-words: environmental crisis; ethics; politics. 